\title{
Blood rheology biomarkers in sickle cell disease
}

\author{
Madeleine Lu', Minke AE Rab², Sergey S Shevkoplyas ${ }^{1}$ and Vivien A Sheehan ${ }^{3}$ (D) \\ ${ }^{1}$ Department of Biomedical Engineering, University of Houston, Houston, TX 77204, USA; ${ }^{2}$ Laboratory of Clinical Chemistry \& \\ Hematology, University Medical Center Utrecht, Utrecht University, Utrecht 3584, The Netherlands; ${ }^{3}$ Department of Pediatrics, Division of \\ Hematology/Oncology, Baylor College of Medicine, Houston, TX 77030, USA \\ Corresponding author: Vivien A Sheehan. Email: vsheehan@bcm.edu
}

\footnotetext{
Impact statement

Severe impairment of blood rheology is the hallmark of SCD pathophysiology, and one of the key factors predisposing SCD

patients to pain crises, organ damage, and early mortality. As novel therapies emerge to treat or cure SCD, it is crucial that these treatments are functionally evaluated for their effect on blood rheology. This review describes a comprehensive panel of rheological biomarkers, their clinical uses, and the technologies used to obtain them. The described technologies can produce highly sensitive measurements of the ability of current treatments to improve blood rheology of SCD patients. The goal of curative therapies should be to achieve blood rheology biomarkers measurements in the range of sickle cell trait individuals (HbAS). The use of the panel of rheological biomarkers proposed in this review could significantly accelerate the development, optimization, and clinical translation of novel therapies for SCD.
}

\begin{abstract}
Sickle cell disease (SCD) is the most common inherited blood disorder, affecting approximately 100,000 patients in the U.S. and millions more worldwide. Patients with SCD experience a wide range of clinical complications, including frequent pain crises, stroke, and early mortality, all originating from a single-point mutation in the $\beta$-globin subunit. The RBC changes resulting from the sickle mutation lead to a host of rheological abnormalities that diminish microvascular blood flow, and produce severe anemia due to RBC hemolysis, and ischemia from vaso-occlusion initiated by sticky, rigid sickle RBCs. While the pathophysiology and mechanisms of SCD have been investigated for many years, therapies to treat the disease are limited. In addition to RBC transfusion, there are only two US Food and Drug Administration (FDA)-approved drugs to ameliorate SCD complications: hydroxyurea (HU) and L-glutamine (Endari ${ }^{\mathrm{TM}}$ ). The only curative therapy currently available is allogeneic hematopoietic stem cell transplantation (HSCT), which is generally reserved for individuals with a matched related donor, comprising only $10-15 \%$ of the total SCD population. Potentially curative advanced gene therapy approaches for SCD are under investigation in ongoing clinical trials. The ultimate goal of any curative treatment should be to repair the hemorheological abnormalities caused by SCD, and thus normalize blood flow and prevent clinical complications. Our mini-review highlights a set of key hemorheological biomarkers
\end{abstract} (and the current and emerging technologies used to measure them) that may be used to guide the development of novel curative and palliative therapies for SCD, and functionally assess outcomes.

Keywords: Sickle cell disease, rheology, gene-based therapy, hydroxyurea, microfluidics

Experimental Biology and Medicine 2020; 245: 155-165. DOI: 10.1177/1535370219900494

\section{Introduction}

SCD is caused by a single missense mutation on the $\beta$-globin chain that changes the amino acid at position 6 from a hydrophilic glutamine amino acid to a hydrophobic valine amino acid. ${ }^{1}$ The most common SCD genotype is the heterozygous HbSS; the compound heterozygous genotype $\operatorname{HbS} \beta^{0}$ (Hemoglobin S-beta thalassemia zero), in which no $\mathrm{HbA}$ is made on the non-sickle mutation beta globin allele, is nearly identical clinically. This review will discuss the hemorheology of these genotypes unless otherwise stated. ${ }^{2}$ Under deoxygenated conditions, HbS polymerizes into a 14-molecule fiber, which subsequently elongates as the result of the interaction of the hydrophobic valine residues with other hydrophobic residues (namely alanine, phenylalanine, and leucine) on the ends of HbS molecules. ${ }^{3}$ Ultimately, these fibers alter the sickle RBC shape, and render the cells more rigid. ${ }^{3}$ Healthy RBCs can squeeze through capillaries as small as $3 \mu \mathrm{m}$, and generally do not adhere to endothelial cells lining the vessels. ${ }^{4}$ In contrast, sickle RBCs impede normal blood flow through direct interactions with the endothelium and other blood components, as well as through the formation of microvascular 
occlusions that promote vasculopathy and ischemic damage ${ }^{5-7}$ Unimpeded passage of RBCs through complex networks of capillaries and larger vessels is crucial for maintaining appropriate level of microvascular perfusion and tissue oxygenation. ${ }^{8}$

\section{Hemorheological abnormalities in SCD}

The clinical complications of SCD can be linked to the abnormal flow properties (blood rheology or hemorheology) of SCD blood. ${ }^{1}$ High viscosity (or thickness) of SCD blood contributes to pain crises and organ damage. Dense cells, defined as having $>1.11 \mathrm{mg} / \mathrm{mL}$ of $\mathrm{Hb}$, are more likely to sickle. Whole blood viscosity and \% dense RBCs (\%DRBC) have been used as classical indicators for the severity of SCD. ${ }^{1,9}$ Blood viscosity is determined by the hematocrit, RBC deformability, RBC aggregation, and plasma viscosity. ${ }^{1,10}$ For a given hematocrit, SCD blood is very viscous in deoxygenated conditions due to HbS sickling: an individual with SCD with a hematocrit of $21 \%$ would have a whole blood viscosity comparable to that of a normal individual with a hematocrit of $45 \%$ even if their blood viscosity is lower than a normal individual's blood viscosity in normoxic conditions. ${ }^{11,12}$ Another way of describing viscosity, the hematocrit to viscosity ratio (HVR), adjusts for differences in hematocrit between individuals, and correlates with oxygen carrying capacity; higher HVR indicates improved oxygen carrying capacity, and it is lower in patients with SCD compared to normal individuals. ${ }^{13}$ In patients with SCD, low HVR was associated with recurrent leg ulcers. ${ }^{1}$ SCD patients with leg ulcers, renal dysfunction, and priapism also have higher \%DRBC and decreased RBC deformability compared to SCD patients without these complications. ${ }^{13,14}$ Therefore, high viscosity and \%DRBC correlate with SCD complications and disease severity, and suggests a role for measurements of RBC rheology in disease and therapy monitoring in patients with SCD.

\section{Senicapoc: A cautionary tale}

Another point to make in discussing the use of hemorheological measurements for assessing SCD therapies is the importance of a multi-modal assessment. Measuring only one aspect of the RBC rheology may miss a change in another important metric that might signal potential adverse events or lack of efficacy. For example, the drug Senicapoc (ICA-17043), a Gardos channel blocker, was investigated to decrease vaso-occlusive crisis (VOC) by reducing RBC dehydration and hemolysis. ${ }^{15}$ Primary endpoints were changes in $\mathrm{Hb}$ level from baseline and \% $\mathrm{DRBC}$, reticulocyte count, and indirect bilirubin were used as secondary end points. While it was shown to decrease hemolysis and was generally considered safe and well tolerated during a phase II clinical trial (NCT00040677), ${ }^{15}$ the drug increased instances of urinary tract infection and nausea with no significant difference in VOC frequency in a phase III clinical trial (NCT000102791), ${ }^{16}$ which was ultimately terminated. It is possible that despite a decline in \% DRBC, whole blood viscosity likely increased with the rise in hemoglobin, which could have led to more instances of VOC. ${ }^{1}$ Additionally, it is known that a decrease in dense and non-deformable RBCs leads to less hemolysis, but not fewer frequencies of VOCs potentially due to high aggregate strength and more adherent RBCs associated with an increase in deformable cells. ${ }^{1,13,17,18}$ The lesson of the Senicapoc trial is that perturbing only one aspect of RBC rheology, even in an apparently favorable direction, may worsen another hemorheological parameter and have a detrimental effect on patient health.

\section{Potential hemorheological biomarkers}

Basic hematological parameters and observation of clinical complications have traditionally served as clinical trial endpoints to assess the effectiveness of new SCD therapies. While incredibly useful, this strategy has some limitations, namely that clinical endpoints, such as the number of pain events, may be difficult to define, subjective, and require a lengthy observation period. Additionally, a change in clinical laboratory values, like the total hemoglobin, may not be consistent with the desired clinical improvement, as the Senicapoc experience suggests. High levels of fetal hemoglobin $(\mathrm{HbF})$ are clearly desirable for patients with $\mathrm{SCD}$. However, it is not known what $\mathrm{HbF}$ level is uniformly consistent with significant clinical improvement in all patients. ${ }^{19}$ Furthermore, pharmacologically induced $\mathrm{HbF}$ is typically unequally distributed throughout the RBC population, resulting in some cells still retaining the capacity to sickle. ${ }^{19}$

Biomarkers that address RBC rheological abnormalities could provide additional essential functional information deepening our understanding of the total effects of novel therapies. Emerging technology such as the Lorrca ${ }^{\circledR}$ ektacytometer with Oxygenscan (RR Mechatronics, Zwaag, The Netherlands) allow the measurement of RBC deformability under a range of oxygen concentrations. \%DRBCs, measured with a phthalate gradient, or an ADVIA hematology analyzer, are present primarily in patients with hemoglobinopathies, and may be higher in patients with more pain events; however, the relationship between \%DRBC and pain is inconsistent, showing different results at different points in the pain cycle, possibly due to increased DRBC destruction during a pain event. ${ }^{20}$ The usage of various microfluidic systems may also provide multiple measures of blood rheology simultaneously: adhesion, deformability, and viscosity. Combining RBC rheological biomarkers with currently used clinical endpoints and standard laboratory testing would ultimately provide a more global picture of the effects of novel therapies on SC patients.

All of these proposed biomarkers require clinical validation. A challenge in developing biomarkers to evaluate clinical interventions is the clinical variability of SCD patients; there may not be an absolute biomarker value indicating improvement, given the variability between patient baselines. Longitudinal monitoring of biomarkers for each patient, capturing pre-intervention baseline values and values while undergoing an intervention may be necessary to assess non-curative therapies. Below, we will describe 
the aspects of SCD pathology that our proposed hemorheological tests will measure.

\section{RBC deformability: Point of sickling, elongation indices}

The normal RBC is a relatively simple structure, as it contains no major organelles, no nucleus, and at rest assumes a biconcave disk shape. ${ }^{21}$ The RBC shape and flexibility allow it to undergo deformations in response to the varying sheer stresses it will experience in large vessels, and the narrow capillaries of the microvasculature. ${ }^{4,8}$ RBCs from individuals with SCD are less deformable compared to RBCs from normal individuals, even when fully oxygenated, when HbS polymerization is less likely to occur. ${ }^{22}$ Sickle RBC deformability declines precipitously with deoxygenation and polymerization.

Currently, deformability is typically measured by ektacytometry and microfiltration techniques. Other techniques such as atomic force microscopy (AFM) and micropipette aspiration can also be employed for single cell analysis although they are generally more time-intensive and require specialized machinery and skilled workers to perform the measurements. ${ }^{23-25}$ Microfiltration techniques measure how fast a set volume of a dilute RBC suspension passes through a membrane with micron size pores. Microfiltration techniques are not standardized across clinics, however so their utility is typically reserved to research settings. ${ }^{25}$ Additionally, microfiltration measurements are highly sensitive to pore plugging (e.g. by residual leukocytes), which may bias the deformability measurements significantly. ${ }^{26}$ In ektacytometry, a small sample of whole blood is mixed with a highly viscous polyvinylpyrrolidone (PVP) solution, and loaded into the space between a rotating cup. A stationary bob is lowered into the rotating cup, forcing the sample up the side of the stationary bob. As the cup rotates, suspended RBCs experience varying shear stresses depending on the speed of the cup rotation. Because the viscosity of PVP solution is higher than that of the $\mathrm{Hb}$ solution inside of the cells, normal, deformable RBCs elongate and orient themselves in response to high shear rates as they would in whole blood even at low hematocrit suspensions. ${ }^{27,28}$ Light from a laser directed at the suspension generates a diffraction pattern from which the elongation index (EI) can be calculated $(\mathrm{EI}=$ (length width)/(length + width)), which is reported as a metric of RBC deformability. It is important to note that ektacytometry provides an average assessment of the hemorheological properties of the RBC population as a whole, not the deformability of individual RBCs as AFM and micropipette aspiration would. ${ }^{29}$

A higher EI indicates greater RBC deformability, and is generally regarded as clinically favorable. This interpretation is supported by the observation that measurements performed on the blood of SCD patients on HU exhibit an increase in RBC deformability after 12 months of therapy (EI increases from $0.14 \pm 0.05$ to $0.22 \pm 0.07$ at $3 \mathrm{~Pa}$ ). ${ }^{30}$ Red cell deformability also improves significantly in patients on chronic RBC transfusions compared to a non-transfused group, likely through the dilution of sickle RBCs with normal RBCs. ${ }^{31}$ Patients with less deformable cells also commonly present with priapism, leg ulcers, and kidney disease potentially brought on by increased hemolysis of the rigid RBCs. ${ }^{18}$ Of note, deformable sickle RBCs have also been shown to be more adherent to vascular endothelial cells, contributing to the onset of crisis events and is also associated with osteonecrosis; therefore, while increased deformability is typically considered favorable, there are still some instances where increased deformability are associated with negative outcomes and care providers should be observant of how deformability plays a role in each individual patient's symptoms. ${ }^{32,33}$

A new addition to the conventional Lorrca ${ }^{\circledR}$, the Oxygenscan, changes the oxygen tension via deoxygenation with nitrogen gas, thereby reducing the oxygen pressure $\left(\mathrm{pO}_{2}\right)$; this allows measurement of $\mathrm{RBC}$ deformability in the oxygenated and deoxygenated state, and identifies the $\mathrm{pO}_{2}$ at which the first $\mathrm{RBCs}$ begin to sickle, termed point of sickling (PoS). ${ }^{22}$ The EI measured when RBCs are oxygenated, and therefore are less likely to have HbS polymers present, corresponds to highest RBC flexibility (EImax). ${ }^{34}$ The EI measured when the RBCs are deoxygenated, at peak $\mathrm{HbS}$ polymerization, corresponds to lowest RBC deformability (EImin) (Figure 1(a)). The EImin, EImax, and PoS vary from individual to individual, and can be changed therapeutically. Figure 1 shows the components of a typical Lorrca $^{\circledR}$ data read-out, sample curves by genotype (Figure 1 (b)), and the impact of $\mathrm{HU}$ and transfusion therapy on EImin, EImax, and PoS (Figure 1(c) and (d)).

\section{RBC ability to perfuse a capillary network}

Artificial microvascular networks (AMVN) are an emerging new class of microfluidic devices and systems that enable analysis of the ability of RBC suspensions to perfuse complex networks of capillary-size microchannels. AMVNs address some of the limitations associated with conventional ektacytometry and the microfiltration assays. A typical AMVN device consists of several networks designed to mimic the various vessel sizes and branching patterns that occur in the microvasculature (Figure 2(a)). RBCs are suspended in buffer or plasma at a physiological hematocrit, then loaded into the AMVN device, and allowed to perfuse the network at a specific driving pressure. The bulk sample flowrate or the flowrate in individual capillaries of the device is calculated using image analysis. ${ }^{35-37}$ In the AMVN, RBCs must undergo a wide range of deformations when passing through the network that may contain channels as large as $70 \mu \mathrm{m}$, and some as small as $3-5 \mu \mathrm{m}$ in width. Any lack in deformability exhibited by RBCs will be observed as an overall decrease in the measured flowrate. In addition to RBC deformability, the AMVN perfusion rate is affected by other hemorheological parameters, including hematocrit, ${ }^{38,39}$ RBC aggregation, ${ }^{39}$ and RBC shape. ${ }^{40}$

Abnormal adhesion of sickle RBCs to vessel walls also plays an important role in obstructing blood flow in SCD patients. The shape change of sickle RBCs changes the normal flow patterns and encourages RBC interactions with endothelial cells. Additionally, all components of 

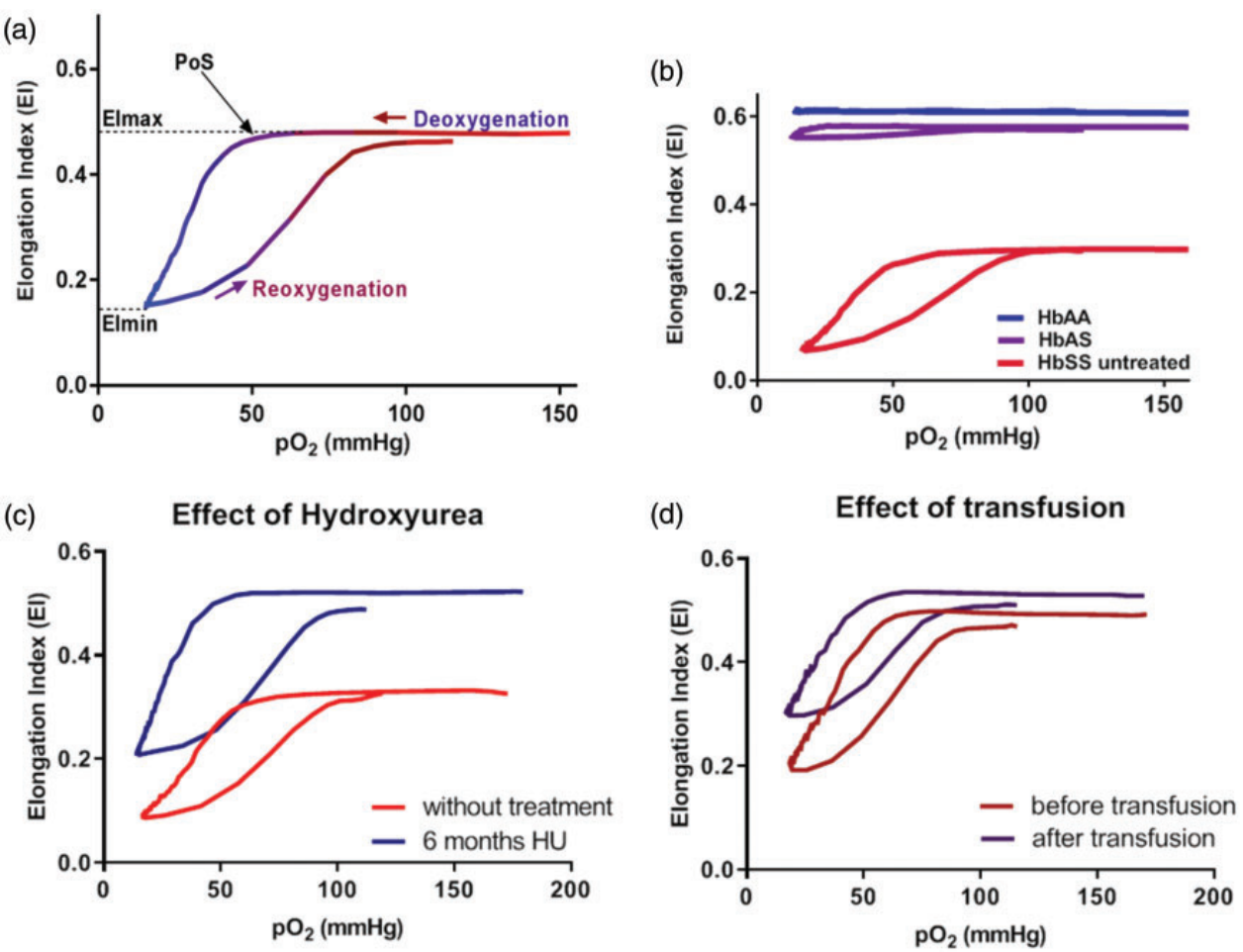

Figure 1. Sample data from Lorrca ${ }^{\circledR}$ with Oxygenscan. (a) Elmax indicates deformability under oxygenated conditions; Elmin under deoxygenated conditions; PoS is the oxygen concentration at which deformability declines. (b) $\mathrm{HbAA} R B C s$ are unaffected by hypoxia while $\mathrm{HbAS}$ shows a slight reduction in deformability at low pO ${ }_{2}$ and HbSS RBCs deformability declines precipitously with hypoxia. (c,d) Hydroxyurea and transfusion improve deformability of the RBC populations. ${ }^{22}$ (A color version of this figure is available in the online journal.)

blood flow in SCD-RBCs, white blood cells (WBCs), and endothelial cells-express pro-adhesion markers. Examples are Lu/BCAM and phosphatidylserine (PS) on RBCs, ${ }^{41,42}$ P-selectin on activated platelets, ${ }^{43}$ and L-selectin on WBCs. ${ }^{44}$ These adhesion molecules interact with exposed sub-endothelial laminin and fibronectin on the surface of endothelial cells lining the vasculature. ${ }^{45}$ This increased adhesion promotes vaso-occlusion in SCD, as it slows blood flow, leading to deoxygenation of the RBC and potentiating sickling. Even RBCs with normal deformability, but increased adhesiveness, increase vascular resistance by $35 \%$ compared to the control group. ${ }^{46}$ This exemplifies how altering one aspect of SCD pathophysiology, such as improving deformability, may not be sufficient if adhesion remains unchanged.

The AMVN devices, as well as other types of microfluidic technologies, ${ }^{47-51}$ can be coated in relevant adhesion molecules to observe the differences in flow rate between non-adhesive and adhesive networks, to better model the in vivo environment (Figure 2(b)). These protein-lined devices also allow for the concurrent assessment of the effect of both deformability, by examining flow through capillarysized channels, and adhesiveness, through adhesion molecules lining the channel walls, on microvascular blood flow dynamics (unpublished from the Shevkoplyas Lab). Importantly, the AMVN perfusion measurements are performed using RBC samples at physiological hematocrits, which accounts for the influence of sub-populations (permanently sickled and/or DRBC), and verifies that deformability of both adherent and non-adherent cells contributes to the measurements.

\section{RBC morphology}

Sickle RBCs exhibit a high level of morphological heterogeneity at the single-cell level. Therefore, it is important to observe and monitor RBC shape at the single-cell level, as well as in bulk volumes, like the AMVN and Lorrca ${ }^{\circledR}$ readouts. In SCD, single cell morphology has traditionally been examined using a simple peripheral blood smear made under hypoxic conditions to potentiate sickling. ${ }^{52,53}$ Briefly, whole blood can be incubated at a low oxygen tension, fixed and preserved on a slide, and then observed under a microscope to quantify sickled RBCs in hypoxic conditions. This method typically relies on an expert observer to manually count the individual cells, which can be prone to human error and limits the sample size that can be feasibly counted. Moreover, these protocols can also be laborious and highly dependent on the skill of the operator, making them difficult to standardize across multiple centers. Therefore, quantifying via blood smear is typically only performed in research settings and rarely used in a clinical setting.

Automated classification of RBC morphologies has been performed to investigate and quantify sickled RBCs. Imaging flow cytometry (IFC) has been used to rapidly enumerate sickled RBCs from a sample of RBCs fixed 
(a)
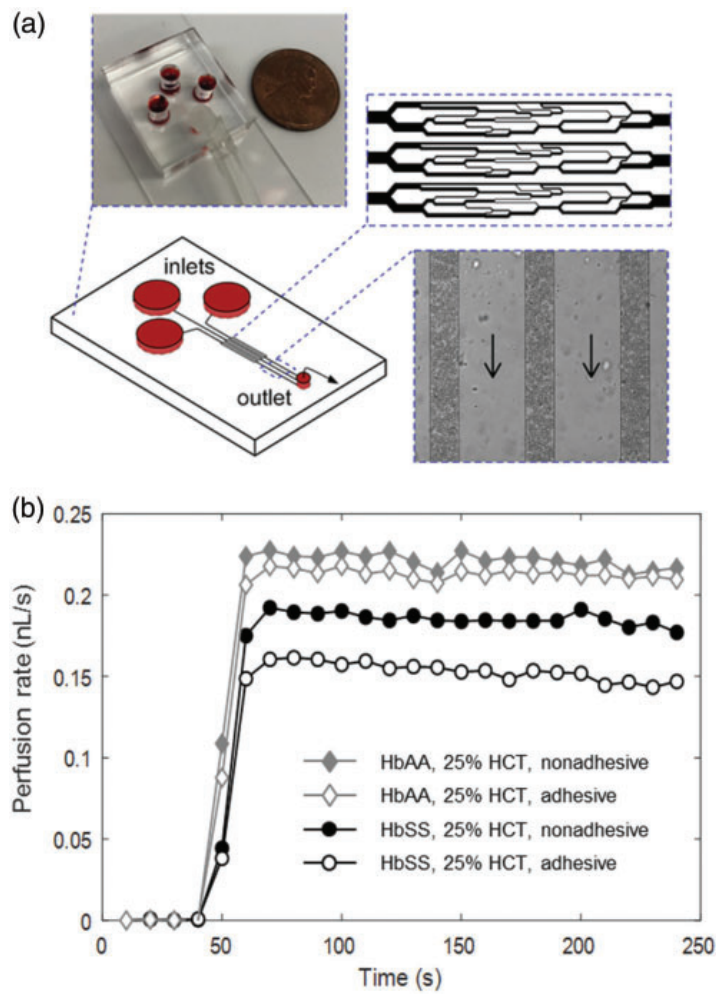

Figure 2. Artificial microvascular network (AMVN). (a) Each AMVN device has three identical capillary network units that meet at a single outlet. Up to three of these network units can be made adhesive using relevant adhesion molecules (e.g. laminin and fibronectin). (b) The perfusion rate of the RBC sample in either an adhesive or a nonadhesive network is determined by image analysis of the RBC flow in the post-capillary venules of the network unites (rightmost inset). Arrows indicate flow direction. (A color version of this figure is available in the online journal.) after being allowed to sickle in hypoxic conditions. ${ }^{54-56}$ To that end, Fertrin et al. further evaluated the concentration of intracellular $\mathrm{HbF}$ on morphology of individual sickle RBCs. ${ }^{57}$ Deoxygenated sickle RBCs are first fluorescently labeled against $\mathrm{HbF}$ and then imaged using IFC which determines the $\mathrm{HbF}$ expression and morphology of the cells. ${ }^{57}$ Automated classification of RBC morphologies can also be accomplished by standardizing acquisition of highresolution microscopic images of individual RBCs using microfluidic devices, and then automatically classifying RBC shape using standard decision-tree-based algorithms or novel approaches based on deep learning (Figure 3). ${ }^{40,58}$ These image analysis techniques can also be applied to a variety of traditional protocols (such as basic peripheral blood smears and sickling assays) as well as emerging microfluidic technologies that investigate RBC sickling behavior under hypoxia. ${ }^{59}$ Similar label-free classification of cell types using machine learning has also been applied to achieve highly accurate identification of $\mathrm{T}$ and $\mathrm{B}$ lymphocytes. ${ }^{60}$ Employing such automated techniques to the identification of irreversible sickled versus normal RBCs would create a more robust, standardized method for evaluating the effect of novel therapies on morphology of individual sickle RBCs.

\section{$\%$ Dense red blood cells}

Sickle RBCs are characteristically denser and more dehydrated than normal RBCs. The traditional approach to measuring \%DRBCs is via the phthalate-gradient method, where two phthalate-oil esters are combined to produce mixtures of precise, known densities. ${ }^{62}$ Washed RBC

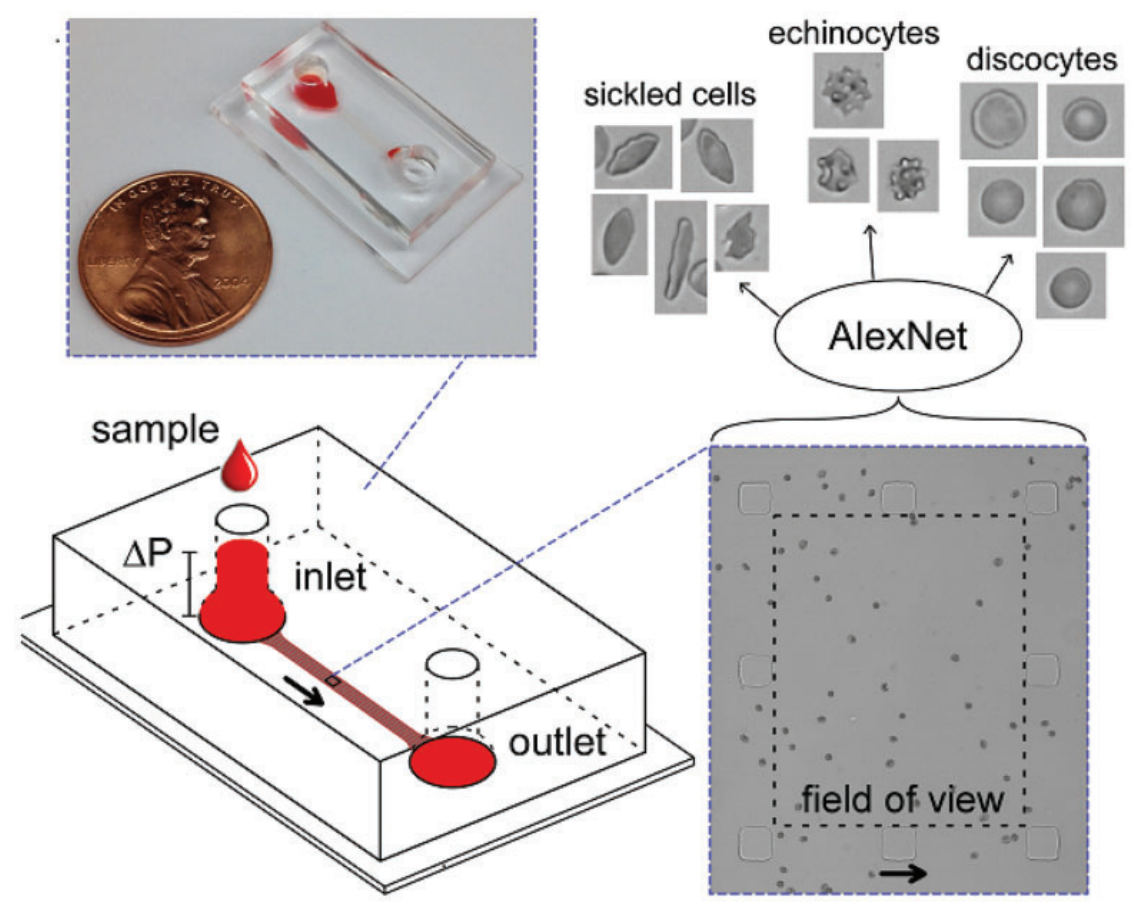

Figure 3. Automated measurement of RBC morphology. A microfluidic device fabricated to allow RBCs to arrange in a single layer under flow is used to acquired multiple images of sickle RBCs. The images are then classified as sickle RBCs, normal RBCs, or RBCs with other aberrant morphologies (e.g. echinocytes) using a pretrained convolutional neural network (AlexNet). Arrows indicate flow direction. ${ }^{61}$ (A color version of this figure is available in the online journal.) 
suspensions are introduced to the mixture, placed into a small glass capillary tube for measuring spun hematocrit, and centrifuged at high speeds. \%DRBCs can be calculated by quantifying the fraction of RBCs located above and below the phthalate oil layer. ${ }^{62}$ Phthalate-gradients require sickle RBCs to undergo multiple rounds of high-speed centrifugation (to wash the RBC samples and to perform the density gradient protocol), which can potentially selectively lyse severely sickled RBC populations and thus effects the measurements. Also, performing these density gradients is a laborious and time-consuming task, making it unattractive to use in a clinical setting. In contrast, the ADVIA hematology analyzer (Siemens, Tarrytown, NY, USA) measures \%DRBCs, defined as the percentage of RBCs with hemoglobin concentration of $41 \mathrm{~g} / \mathrm{dL}$ or higher, using only $200 \mu \mathrm{L}$ of whole blood sample. RBC samples are placed in a hypotonic solution that forces normal RBCs to swell. Sickle RBCs that are unable to become spheres are considered dense cells as they exhibit a high $\mathrm{Hb}$ concentration and a low volume. The ADVIA hematology analyzer is used routinely in the clinical settings, and therefore it can easily be adopted to also measure \% DRBCs on blood samples from SCD patients.

\section{Role of hemorheological biomarkers in evaluation of currently approved therapies for SCD}

Currently, there are only two drugs approved by the FDA for use in SCD: HU and L-glutamine. Both of these drugs have been shown to decrease VOC in patients in clinical trials. ${ }^{63,64} \mathrm{HU}$ ameliorates the complications of SCD by inducing $\mathrm{HbF}$, reducing the $\mathrm{WBC}$ count, reducing the number of adhesive reticulocytes, and reducing inflammation. ${ }^{65,66}$ The HU-induced rise in $\mathrm{HbF}$ helps reduce polymerization of $\mathrm{HbS}$, allowing sickle RBCs to survive longer in the circulation. Patients who respond well to HU, which include most pediatric patients and about half of adult patients, may experience a $1 \mathrm{~g} / \mathrm{dL}$ or higher increase in $\mathrm{Hb}$. While a rise in $\mathrm{Hb}$ typically increases whole blood viscosity, the improvements HU makes to the quality, or rheology of the blood offsets this, and patients do not typically show a rise in whole blood viscosity as measured by a cone and plate viscometer ex vivo, despite a rise in $\mathrm{Hb}^{29}$

HU has also been shown in vitro to decrease sickle RBC adhesion to the endothelium, possibly through a reduction in $\mathrm{Lu} / \mathrm{BCAM}$ phosphorylation. ${ }^{41,67,68}$ Our own studies, however, show that perfusion rate through an adhesive AMVN for sickle RBCs does not reach the same level as for normal RBCs, either for patients on HU or chronic transfusion therapy. Interestingly, even with no significant change in $\% \mathrm{HbF}$, $\mathrm{HU}$-treated patients were shown to have decreased \%DRBCs after six months post-treatment. ${ }^{14} \mathrm{HU}$ also has been reported to decrease the number of irreversibly sickled RBCs. ${ }^{69}$ Therefore, patients may still benefit from $\mathrm{HU}$-therapy even without significant $\mathrm{HbF}$ induction if other hemorheological benefits are achieved, such as reduced RBC adhesiveness, ${ }^{41,67,68}$ reduced RBC hemolysis associated with less dense cells, ${ }^{17}$ and decreased robustness of RBCs aggregates. ${ }^{20,29,32}$
A pivotal phase III clinical study (NCT01179217) conducted by Emmaus Medical, Inc. showed that L-glutamine resulted in a lower incidence of VOC as well as a lower rate of hospitalizations and shorter hospital stays. ${ }^{70}$ However, no changes in standard clinical laboratory values were noted. ${ }^{71}$ The clinical improvements were believed to be due to an increase in the proportion of the reduced form of nicotinamide adenine dinucleotides in the RBCs of SCD patients, which reduces the oxidative stress. While the endpoints in the phase III study were clinically important, it is essential that quantitative, objective biomarkers or measurable laboratory changes be identified that can serve as endpoints for future clinical trials assessing dose optimization and the efficacy and safety of L-glutamine in SCD individuals, including those with hepatic and renal dysfunction. As stated, high whole blood viscosity, high $\%$ DRBCs, and adherent RBCs are associated with higher rates of VOC. Given the demonstrated reduction in pain events, L-glutamine may be improving blood rheology by altering an intrinsic property of the RBCs; it may also act by reducing $\mathrm{RBC}$ adherence to the endothelium, often the sentinel event preceding a VOC. For instance, sickle RBC adhesion to endothelial cells has been shown to decrease after patients received oral L-glutamine as treatment. ${ }^{70}$ However, it is important to note that these studies were performed using static adhesion assays where RBCs were allowed to sediment onto endothelial cell-coated wells before removing non-adherent RBCs via aspiration, or by inverting the well-plate. ${ }^{70}$ These methods do not accurately replicate adhesion of sickle RBCs under the physiological conditions in vivo, which occur under flow, in the presence of cell-to-cell interactions and while cells navigate the confined geometries of networks of microvessels. Moreover, while the morphology of SCD RBCs was reported to have generally improved with L-glutamine therapy, this appears to be a qualitative observation by the authors. L-glutamine did not improve the ability of deoxygenated sickle RBCs to pass through uniform sieves (RBC filterability), a more objective assessment of deformability. ${ }^{71}$ More sophisticated testing of L-glutamine's effects on hemorheological properties like RBC adhesiveness and deformability using novel microfluidic tools such as the AVMN and the deeplearning-enabled morphological assays are needed to validate this therapy's efficacy and mechanism of action. Assessment of L-glutamine's effects using the Lorrca ${ }^{\circledR}$ with Oxygenscan will allow evaluation of deformability under both oxygenated and deoxygenated conditions, as well as a determination of the oxygen tension at which sickling occurs, the PoS. Such a comprehensive panel of hemorheological tests could help address concerns about the efficacy and mechanism of L-glutamine, and provide an objective quantitative biomarker for use in future clinical trials or to monitor patient response.

Allogeneic hematopoietic stem cell transplantation (allo-HSCT) is the only curative treatment option currently available for SCD, and under the most ideal conditions, allo-HSCT patients have an overall survival rate higher than $90 \% .{ }^{72}$ However, the need for a matched donor limits availability of allo-HSCT to only $10-15 \%$ of the SCD patient population, ${ }^{73}$ and various complications can 
occur post-transplantation including: graft vs. host disease (GVHD), graft rejections, possible sterility, and HSCTrelated organ toxicities. Furthermore, while allo-HSCT is typically reserved for patients with severe $\mathrm{SCD}$, successful HSCT is also dependent on recipients being at good functional baseline and without irreversible organ damage prior to transplantation, further limiting the prospective patient population. ${ }^{73}$ If at baseline, patients already suffer from irreversible organ damage, then even successful matched-donor allo-HSCT can still have irregular blood flow. Therefore, patients should be closely monitored post-transplantation for signs of hemolysis and indications of abnormal hemorheology. Morphological analysis can assess whether or not organ damage, particularly damage typically seen in SCD like splenic damage, affects the quality of circulating RBCs. ${ }^{74}$ Even if patients present with reduced pain events, they can still remain at risk of SCD-related organ damage and early mortality if hemolysis continues. A comprehensive panel of hemorheological tests periodically assessed following allo-HSCT would help monitor the long-term efficacy of this therapy.

\section{Role of hemorheological biomarkers in development of novel therapeutic treatments for SCD}

Novel therapeutic approaches for mitigating the symptoms of SCD and improving clinical outcomes can be categorized based on the SCD pathology they are designed to target. One such class is $\mathrm{Hb}$ modifiers; the intent being to modify the $\mathrm{HbS}$ so it cannot sickle. A promising example is Voxelotor (GBT440-007; Global Blood Therapeutics, San Francisco, CA, USA), a small molecule that modifies $\mathrm{HbS}$ by stabilizing the R-state of $\mathrm{Hb}$ conformation, increasing $\mathrm{Hb}$ oxygen affinity and reducing polymerization. ${ }^{75}$ A phase I/II clinical trial for Voxelotor showed it was well tolerated and had a dose-dependent increase in oxygen-affinity. ${ }^{76}$ Adolescents receiving Voxelotor had an increase of $1.0 \mathrm{~g} / \mathrm{dL}$ in their $\mathrm{Hb}$ level and a reduction of both reticulocytes and indirect bilirubin, indicating a reduction in sickle RBC hemolysis (NCT02285088). ${ }^{77}$ A phase III clinical trial investigating both safety and efficacy of Voxelotor in adults found Voxelotor significantly increased $\mathrm{Hb}$ levels and reduced markers for hemolysis for compared to placebo (NCT03036813). ${ }^{77}$ Patients receiving Voxelotor had a sustained decrease of approximately $70 \%$ in the number of irreversibly sickled RBCs after 90 days of treatment. ${ }^{78}$ Because of these positive findings, the FDA designated Voxelotor as a "breakthrough therapy" and granted it priority review. Voxelotor also improved sickle RBC deformability as observed using micropipette and filtration techniques and blood viscosity in vitro.$^{79}$ However, no tests of the effect of Voxelotor on blood viscosity in vivo have been reported. $\mathrm{Hb}$ rise typically increases blood viscosity, which can be deleterious to patients with SCD by promoting VOC, as seen with Senicapoc. ${ }^{1}$ However, it seems likely that when Voxelotor is present and bound to at least $30 \%$ of the $\mathrm{Hb}$ molecules, $\mathrm{HbS}$ is less likely to become deoxygenated and sickle, analogous to $\mathrm{HbF}^{80}$ Of greater concern is viscosity rise in patients who stop taking
Voxelotor after experiencing a rise in $\mathrm{Hb}$. Unlike $\mathrm{HbF}$, the $\mathrm{HbS}$ previously bound to Voxelotor will revert back to its original state within hours, with a concomitant decrease in oxygen affinity. The greater amounts of unmodified $\mathrm{HbS}$ may significantly increase blood viscosity until the extra $\mathrm{HbS}$ is removed through hemolysis in 14-20 days. If viscosity does rise when Voxelotor is stopped, this would not invalidate this potentially very useful drug; it would instead prompt anticipatory guidance of patients to avoid sudden cessation, and consideration of a drug wean. However, an increase in deformability without the decrease in RBC adherence could contribute to the onset of VOCs via direct RBCs to endothelial interactions and increased RBC aggregation. Studies on the adhesive properties of RBCs of Voxelotor-treated RBCs would be pertinent to investigate the potential for these events to occur. Morphology analysis would also be an important metric to attain as less $\mathrm{HbS}$ polymerization should lead to fewer irreversibly sickled RBCs. Moreover, single cell analysis on deformability using techniques like AFM and micropipette can also quantify to what extent Voxelotor inhibition of HbS polymerization affects RBC deformability.

Another drug of interest is Crizanlizumab (SEG101; Novartis, Basal, Switzerland), which has also been granted priority review by the FDA for use in SCD. Crizanlizumab is a P-selectin monoclonal antibody that blocks interaction of P-selectins with leukocytes. A 12-month long clinical trial (SUSTAIN Trial; NCT01895361) assessed the safety and efficacy of Crizanlizumab for patients both on and off HU therapy. Treatment with high-dose Crizanlizumab resulted in reduced rates of VOC compared to placebo, and increased the time to the first crisis. ${ }^{43}$ No significant changes in $\mathrm{Hb}$, lactate dehydrogenase, reticulocyte count, and indirect bilirubin were observed between Crizanlizumab and placebo groups. ${ }^{43}$ Therefore, it seems that the benefits of Crizanlizumab are not related to intrinsic RBC changes or inhibition of hemolysis. At least four more clinical studies are either recruiting or currently active to investigate dose confirmation and safety with primary end points being frequency of adverse events and organ damage. It would be advantageous to study how Crizanlizumab and similar agents affect blood flow through the adhesive AMVN technologies, considering the main target is the prevention of adhesive events. AMVN technologies and other adhesion flow devices that run whole blood can easily investigate the efficacy of the drug by directly analyzing leukocyte rolling on devices coated with P-selectins. ${ }^{81}$

\section{Role of hemorheological biomarkers in the development of potentially curative gene therapies for SCD}

Cellular- and gene-based therapies are an area of intense investigation; they can provide means to extend stem cell transplant to a larger number of SCD patients, as the patient is their own donor. Hematopoietic stem and progenitor cells are harvested from the patient, modified using lentiviruses or genome editing, and transplanted back into the patient. Currently, there are a small number of clinical trials 
Table 1. Typical ranges attained by the Sheehan and Shevkoplyas Labs for the panel of propose rheological biomarkers.

\begin{tabular}{|c|c|c|c|c|c|c|c|c|c|}
\hline Biomarkers & \multicolumn{3}{|l|}{ HbAA } & \multicolumn{3}{|l|}{ HbAS } & \multicolumn{3}{|l|}{ HbSS } \\
\hline PoS (mmHg) & $\mathrm{N} / \mathrm{A}$ & $\mathrm{N} / \mathrm{A}$ & $\mathrm{N} / \mathrm{A}$ & $5.0-12.1$ & 6.7 & 2.4 & $17.0-85.4$ & 41.7 & 9.961 \\
\hline Elmax & $0.6-0.62$ & 0.61 & 0.006 & $0.6-0.63$ & 0.61 & 0.12 & $0.22-0.59$ & 0.48 & 0.082 \\
\hline Elmin & $\mathrm{N} / \mathrm{A}$ & $\mathrm{N} / \mathrm{A}$ & $\mathrm{N} / \mathrm{A}$ & $0.54-0.60$ & 0.58 & 0.21 & $0.04-0.5$ & 0.14 & 0.096 \\
\hline$\%$ DRBC & $0.1-1.1$ & 0.7 & 0.6 & $0.1-1.1$ & 0.6 & 0.5 & $2.0-21.5$ & 4.8 & 3.5 \\
\hline AMVN Adhesive (nL/s) & $0.21-0.23$ & 0.21 & 0.01 & $\mathrm{~N} / \mathrm{A}$ & $\mathrm{N} / \mathrm{A}$ & $\mathrm{N} / \mathrm{A}$ & $0.14-0.18$ & 0.15 & 0.02 \\
\hline
\end{tabular}

The $\mathrm{HbSS}$ ranges for this proposed panel do not overlap either $\mathrm{HbAA}$ or $\mathrm{HbAS}$ ranges indicating the sensitivity of each test to differentiate between $\mathrm{HbSS}$ with $\mathrm{HbAS}$ and $\mathrm{HbAA}$. RBC morphology is presented as percentage of RBCs with irregular morphologies.

studying viable gene therapy (lentivirus based) and gene editing (CRISPR/Cas9 based) approaches.

Lentivirus-based (LV) gene therapies have been used to treat patients with $\beta$-hemoglobinopathies, with a number of clinical trials performed around the world. For SCD, LV-based therapies have two primary aims: increase the induction of $\mathrm{HbF}$ by introducing LVs containing a $\gamma$ - or a hybrid $\beta$-promotor $/ \gamma$-globin gene, or by introducing mutated $\beta$-globin that interferes with $\mathrm{HbS}$ and prevents polymerization. $^{82}$ The BB305 LentiGlobin (Bluebird Bio, Cambridge, MA) is a self-inactivating lentivirus developed to encode an "anti-sickling" $\beta$-globin. A published report of LentiGlobin gene-based therapy in a 13-year-old with SCD showed approximately $47 \% \mathrm{HbA}^{\mathrm{T} 87 \mathrm{Q}}$ and $2 \% \mathrm{HbF}$, and total $\mathrm{Hb}$ of $11.7 \mathrm{~g} / \mathrm{dL}^{83}$

Other gene editing therapy strategies include like zinc finger nucleases, transcription activator-like effector nucleases, and clustered regulatory interspaced short palindromic repeats and associated Cas9 (CRISPR/Cas9). CRISPR/Cas 9 system shows a promising editing rate of 85-90\% after 16 weeks in human bone marrow cells in preclinical studies. ${ }^{84}$ CRISPR Therapeutics and Vertex Pharmaceuticals are currently recruiting participants with severe SCD for a clinical trial (NCT03745287) to be treated with their novel CRISPR/Cas9 technology CTX001.

The challenges of these gene-based therapies are significant: potential off-target effects, the need for high levels of correction, and need for effective engraftment. An additional challenge is that we do not know what level of correction of the sickle mutation or induction of $\mathrm{HbF}$ is curative. Therefore, a comprehensive functional analysis of the resulting blood, after a patient has undergone gene-based therapy, is essential for further development of these novel therapies, and verification that a cure has been achieved. In order to consider a gene-based therapy as curative, the patient should have rheology that has been normalized to at least that of sickle cell trait (SCT) patient, who are considered to have a relatively benign form of SCD when compared to the HbSS variant. Clinically, this will be observed as stiffened, less deformable RBCs in reduced $\mathrm{pH}$ solutions, but with few if any sickling events in deoxygenated conditions. Exercise-induced increases in epinephrine could contribute to increased adhesion of both SCD and SCT RBCs and should be investigated for gene-edited RBCs as well. ${ }^{59,85}$
A battery of hemorheological tests reviewed in this article-RBC deformability in oxygenated and deoxygenated conditions, perfusion of adhesive AMVN, $\%$ DRBC and RBC morphology-are well-suited for comprehensive evaluation of whether or not a cure has been achieved. Table 1 shows typical ranges of values for the proposed battery of hemorheological tests for individuals with $\mathrm{HbAA}, \mathrm{HbAS}$, and HbSS genotypes. There is no overlap between $\mathrm{HbAS}$ and HbSS for the values of EImin, EImax, and PoS measured using Lorrca ${ }^{\circledR}$ with Oxygenscan. Since HbAA does not sickle, there is only EImax, and no PoS; HbAS blood sickles minimally at very low oxygen concentrations. Of note, the HbSS biomarker values are from patients 1 to 21 years of age, on $H U$, and chronic transfusion. While these therapies ameliorate the symptoms of SCD, and some patients appear asymptomatic, they do not constitute a cure, and none fall within the range of $\mathrm{HbAS}$. Whole blood viscosity measured $45 \mathrm{~s}^{-1}$ and $225 \mathrm{~s}^{-1}$ at $37^{\circ} \mathrm{C}$, and the HVR values calculated using these values showed significant overlap between the genotypes, suggesting that viscosity and HVR cannot distinguish between cured and not cured states. \%DRBCs, as measured by ADVIA, can distinguish between HbSS and HbAS, although the overlap between $\mathrm{HbAA}$ and $\mathrm{HbAS}$ is notable. Additional work is needed to further develop the adhesive AMVN and morphology technologies and properly define the reference ranges for each genotype. Another challenge is the needed to strictly use the international guidelines published on blood rheology and the specific guidelines published in the field of SCD, in order to generate data comparable across clinical sites and laboratories.

\section{Conclusion}

As we continue to expand the therapeutic and curative treatment options for SCD, hemorheological biomarkers serve as an important metric for analyzing the overall quality of the treatment. Reproducible assays that have a strong association with SCD-related clinical complications should also be incorporated into clinical trials to ensure a complete picture of the effects of the novel therapies is being seen. The goal of any novel SCD therapy should be to significantly improve or normalize blood rheology. Measurements of deformability, point of sickling, red cell density and adhesion provide information about RBC function beyond that of standard laboratory values such as $\% \mathrm{HbF}, \mathrm{CBC}$, 
reticulocyte count, and markers of hemolysis. Hemorheological biomarkers must be evaluated in therapies with curative intent; gene-based therapies that do not achieve the hemorheological correction comparable to $\mathrm{HbAS}$ should be optimized further.

Authors' contributions: ML, SSS and VAS wrote the manuscript. MAER contributed Figure 1 and edited the manuscript.

\section{DECLARATION OF CONFLICTING INTERESTS}

VAS performs research supported by funds from Emmaus Medical, Inc. and RR Mechatronics. MAER performs research supported by RR Mechatronics. SSS is an inventor of some of the technology discussed in this review; he has received compensation as consultant, and research funding from New Health Sciences, Inc. (d/b/a Hemanext) which is commercializing the artificial microvascular network technology.

\section{FUNDING}

National Institutes of Health, National Institute of Diabetes and Digestive and Kidney Diseases grant 1K08DK110448-01, research grants from Emmaus Medical, Inc. (VAS) and RR Mechatronics (VAS, MAER).

\section{ORCID iD}

Vivien A Sheehan (iD https://orcid.org/0000-0003-2837-2255

\section{REFERENCES}

1. Connes P, Alexy T, Detterich J, Romana M, Hardy-Dessources MD, Ballas SK. The role of blood rheology in sickle cell disease. Blood Rev 2016;30:111-8

2. Hassell KL. Population estimates of sickle cell disease in the US. Am J Prev Med 2010;38:S512-21

3. Vekilov PG. Sickle-cell haemoglobin polymerization: is it the primary pathogenic event of sickle-cell anaemia? Br J Haematol 2007;139:173-84

4. Mohandas N, Gallagher PG. Red cell membrane: past, present, and future. Blood 2008;112:3939-48

5. Matsui NM, Borsig L, Rosen SD, Yaghmai M, Varki A, Embury SH. Pselectin mediates the adhesion of sickle erythrocytes to the endothelium. Blood 2001;98:1955-62

6. Goel MS, Diamond SL. Adhesion of normal erythrocytes at depressed venous shear rates to activated neutrophils, activated platelets, and fibrin polymerized from plasma. Blood 2002;100:3797-803

7. Byrnes JR, Wolberg AS. Red blood cells in thrombosis. Blood 2017;130:1795-9

8. Lipowsky HH. Microvascular rheology and hemodynamics. Microcirculation 2005;12:5-15

9. Nader E, Skinner S, Romana M, Fort R, Lemonne N, Guillot N, Gauthier A, Antoine-Jonville S, Renoux C, Hardy-Dessources MD, Stauffer E, Joly P, Bertrand Y, Connes P. Blood rheology: key parameters, impact on blood flow, role in sickle cell disease and effects of exercise. Front Physiol 2019;10:e1329

10. Baskurt OK, Meiselman HJ. Blood rheology and hemodynamics. Semin Thromb Hemost 2003;29:435-50

11. Li X, Du E, Lei H, Tang YH, Dao M, Suresh S, Karniadakis GE. Patientspecific blood rheology in sickle-cell anaemia. Interface Focus 2016;6: e20150065

12. Renoux C, Romana M, Joly P, Ferdinand S, Faes C, Lemonne N, Skinner S, Garnier N, Etienne-Julan M, Bertrand Y, Petras M, Cannas G, Divialle-Doumdo L, Nader E, Cuzzubbo D, Lamarre Y, Gauthier A, Waltz X, Kebaili K, Martin C, Hot A, Hardy-Dessources MD, Pialoux
V, Connes P. Effect of age on blood rheology in sickle cell anaemia and sickle cell haemoglobin C disease: a cross-sectional study. PLoS One 2016;11:e0158182

13. Connes P, Lamarre Y, Hardy-Dessources MD, Lemonne N, Waltz X, Mougenel D, Mukisi-Mukaza M, Lalanne-Mistrih ML, Tarer V, Tressieres B, Etienne-Julan M, Romana M. Decreased hematocrit-toviscosity ratio and increased lactate dehydrogenase level in patients with sickle cell anemia and recurrent leg ulcers. PLoS One 2013;8:e79680

14. Bartolucci P, Brugnara C, Teixeira-Pinto A, Pissard S, Moradkhani K, Jouault H, Galacteros F. Erythrocyte density in sickle cell syndromes is associated with specific clinical manifestations and hemolysis. Blood 2012;120:3136-41

15. Ataga KI, Smith WR, De Castro LM, Swerdlow P, Saunthararajah Y, Castro O, Vichinsky E, Kutlar A, Orringer EP, Rigdon GC, Stocker JW; Investigators ICA. Efficacy and safety of the gardos channel blocker, senicapoc (Ica-17043), in patients with sickle cell anemia. Blood 2008;111:3991-7

16. Ataga KI, Reid M, Ballas SK, Yasin Z, Bigelow C, James LS, Smith WR, Galacteros F, Kutlar A, Hull JH, Stocker JW; Investigators ICAS. Improvements in haemolysis and indicators of erythrocyte survival do not correlate with acute vaso-occlusive crises in patients with sickle cell disease: a phase III randomized, placebo-controlled, double-blind study of the gardos channel blocker senicapoc (Ica-17043). Br J Haematol 2011;153:92-104

17. Joiner $\mathrm{CH}$. Gardos pathway to sickle cell therapies? Blood 2008;111:3918-9

18. Connes P, Lamarre Y, Waltz X, Ballas SK, Lemonne N, Etienne-Julan M, Hue O, Hardy-Dessources MD, Romana M. Haemolysis and abnormal haemorheology in sickle cell anaemia. Br J Haematol 2014;165:564-72

19. Steinberg MH, Chui DH, Dover GJ, Sebastiani P, Alsultan A. Fetal hemoglobin in sickle cell anemia: a glass half full? Blood 2014;123:481-5

20. Ballas SK, Smith ED. Red blood cell changes during the evolution of the sickle cell painful crisis. Blood 1992;79:2154-63

21. Geekiyanage NM, Balanant MA, Sauret E, Saha S, Flower R, Lim CT, $\mathrm{Gu}$ Y. A coarse-grained red blood cell membrane model to study stomatocyte-discocyte-echinocyte morphologies. PLoS One 2019;14: e0215447

22. Rab MAE, van Oirschot BA, Bos J, Merkx TH, van Wesel ACW Abdulmalik O, Safo MK, Versluijs BA, Houwing ME, Cnossen MH, Riedl J, Schutgens REG, Pasterkamp G, Bartels M, van Beers EJ, van Wijk R. Rapid and reproducible characterization of sickling during automated deoxygenation in sickle cell disease patients. Am J Hematol 2019;94:575-84

23. Maciaszek JL, Lykotrafitis G. Sickle cell trait human erythrocytes are significantly stiffer than normal. J Biomech 2011;44:657-61

24. Byun H, Hillman TR, Higgins JM, Diez-Silva M, Peng Z, Dao M, Dasari RR, Suresh S, Park Y. Optical measurement of biomechanical properties of individual erythrocytes from a sickle cell patient. Acta Biomater 2012;8:4130-8

25. Huisjes R, Bogdanova A, van Solinge WW, Schiffelers RM, Kaestner L, Van Wijk R. Squeezing for life-properties of red blood cell deformability. Front Physiol 2018;9:e656

26. Sosa JM, Nielsen ND, Vignes SM, Chen TG, Shevkoplyas SS. The relationship between red blood cell deformability metrics and perfusion of an artificial microvascular network. Clin Hemorheol Microcirc 2014;57:275-89

27. Fischer TM, Stohr-Lissen M, Schmid-Schonbein H. The red cell as a fluid droplet: tank tread-like motion of the human erythrocyte membrane in shear flow. Science 1978;202:894-6

28. Lanotte L, Mauer J, Mendez S, Fedosov DA, Fromental J-M, Claveria V, Nicoud F, Gompper G, Abkarian M. Red cells' dynamic morphologies govern blood shear thinning under microcirculatory flow conditions. Proc Natl Acad Sci USA 2016;113:13289-94

29. Lemonne N, Charlot K, Waltz X, Ballas SK, Lamarre Y, Lee K, Hierso R, Connes C, Etienne-Julan M, Romana M. Hydroxyurea treatment does not increase blood viscosity and improves red blood cell rheology in sickle cell anemia. Haematologica 2015;100:e383-86

30. Simmonds MJ, Suriany S, Ponce D, Detterich JA. Red blood cell mechanical sensitivity improves in patients with sickle cell disease 
undergoing chronic transfusion after prolonged, subhemolytic shear exposure. Transfusion 2018;58:2788-96

31. Parrow NL, Violet PC, Tu H, Nichols J, Pittman CA, Fitzhugh C, Fleming RE, Mohandas N, Tisdale JF, Levine M. Measuring deformability and red cell heterogeneity in blood by ektacytometry. J Vis Exp 2018;e56910

32. Lemonne N, Lamarre $\mathrm{Y}$, Romana M, Mukisi-Mukaza M, HardyDessources M-D, Tarer V, Mougenel D, Waltz X, Tressières B, Lalanne-Mistrih M-L. Does increased red blood cell deformability raise the risk for osteonecrosis in sickle cell anemia? Blood 2013;121:3054-6

33. Mohandas N, Evans E. Adherence of sickle erythrocytes to vascular endothelial-cells - requirement for both cell-membrane changes and plasma factors. Blood 1984;64:282-7

34. Rab MAE, Kanne CK, Bos J, Boisson C, van Oirschot BA, Nader E, Renoux C, Joly P, Fort R, van Beers EJ, Sheehan VA, van Wijk R, Connes P. Methodological aspects of the oxygenscan in sickle cell disease: a need for standardization. Am J Hematol 2019;95:E5-8

35. Burns JM, Yang X, Forouzan O, Sosa JM, Shevkoplyas SS. Artificial microvascular network: a new tool for measuring rheologic properties of stored red blood cells. Transfusion 2012;52:1010-23

36. Burns JM, Yoshida T, Dumont LJ, Yang X, Piety NZ, Shevkoplyas SS. Deterioration of red blood cell mechanical properties is reduced in anaerobic storage. Blood Transfus-Italy 2016;14:80-8

37. Forouzan O, Yang X, Sosa JM, Burns JM, Shevkoplyas SS. Spontaneous oscillations of capillary blood flow in artificial microvascular networks. Microvasc Res 2012;84:123-32

38. Reinhart WH, Piety NZ, Shevkoplyas SS. Influence of feeding hematocrit and perfusion pressure on hematocrit reduction (fåhraeus effect) in an artificial microvascular network. Microcirculation 2017;24:e12396

39. Reinhart WH, Piety NZ, Shevkoplyas SS. Influence of red blood cell aggregation on perfusion of an artificial microvascular network. Microcirculation 2017;24:e12317

40. Piety NZ, Reinhart WH, Pourreau PH, Abidi R, Shevkoplyas SS. Shape matters: the effect of red blood cell shape on perfusion of an artificial microvascular network. Transfusion 2016;56:844-51

41. Chaar V, Laurance S, Lapoumeroulie C, Cochet S, De Grandis M, Colin Y, Elion J, Le Van Kim C, El Nemer W. Hydroxycarbamide decreases sickle reticulocyte adhesion to resting endothelium by inhibiting endothelial lutheran/basal cell adhesion molecule ( $\mathrm{Lu} / \mathrm{BCAM})$ through phosphodiesterase 4A activation. J Biol Chem 2014;289:11512-21

42. Hannemann A, Rees DC, Brewin JN, Noe A, Low B, Gibson JS. Oxidative stress and phosphatidylserine exposure in red cells from patients with sickle cell anaemia. Br J Haematol 2018;182:567-78

43. Ataga KI, Kutlar A, Kanter J, Liles D, Cancado R, Friedrisch J, Guthrie TH, Knight-Madden J, Alvarez OA, Gordeuk VR, Gualandro S, Colella MP, Smith WR, Rollins SA, Stocker JW, Rother RP. Crizanlizumab for the prevention of pain crises in sickle cell disease. $N$ Engl J Med 2017;376:429-39

44. Al Najjar S, Adam S, Ahmed N, Qari M. Markers of endothelial dysfunction and leucocyte activation in Saudi and non-Saudi haplotypes of sickle cell disease. Ann Hematol 2017;96:141-6

45. Damanhouri GA, Jarullah J, Marouf S, Hindawi SI, Mushtaq G, Kamal MA. Clinical biomarkers in sickle cell disease. Saudi J Biol Sci 2015;22:24-31

46. Kaul DK, Koshkaryev A, Artmann G, Barshtein G, Yedgar S. Additive effect of red blood cell rigidity and adherence to endothelial cells in inducing vascular resistance. Am J Physiol Heart Circ Physiol 2008;295: H1788-93

47. Mannino RG, Myers DR, Ahn B, Wang Y, Margo R, Gole H, Lin AS, Guldberg RE, Giddens DP, Timmins LH, Lam WA. Do-it-yourself in vitro vasculature that recapitulates in vivo geometries for investigating endothelial-blood cell interactions. Sci Rep UK 2015;5:e12401

48. Kim M, Alapan Y, Adhikari A, Little JA, Gurkan UA. Hypoxiaenhanced adhesion of red blood cells in microscale flow. Microcirculation 2017;24:e12374

49. White J, Lancelot M, Sarnaik S, Hines P. Increased erythrocyte adhesion to VCAM-1 during pulsatile flow: application of a microfluidic flow adhesion bioassay. Clin Hemorheol Microcirc 2015;60:201-13
50. Alapan Y, Kim C, Adhikari A, Gray KE, Gurkan-Cavusoglu E, Little JA, Gurkan UA. Sickle cell disease biochip: a functional red blood cell adhesion assay for monitoring sickle cell disease. Transl Res 2016;173:74-91

51. Partola KR, Andemariam B, Lykotrafitis G. Microfluidic experimental setup for adhesion and recovery measurements of red blood cells in sickle cell disease. J Mech Behav Biomed Mater 2017;71:80-4

52. Diakite SAS, Ndour PA, Brousse V, Gay F, Roussel C, Biligui S, Dussiot M, Prendki V, Lopera-Mesa TM, Traore K, Konate D, Doumbia S, Cros J, Dokmak S, Fairhurst RM, Diakite M, Buffet PA. Stage-dependent fate of Plasmodium falciparum-infected red blood cells in the spleen and sickle-cell trait-related protection against malaria. Malaria Journal 2016;15:e482

53. Abay A, Simionato G, Chachanidze R, Bogdanova A, Hertz L, Bianchi $P$, van den Akker E, von Lindern M, Leonetti M, Minetti G. Glutaraldehyde - a subtle tool in the investigation of healthy and pathologic red blood cells. Front Physiol 2019;10:1-14

54. Samsel L, McCoy JP, Jr. Imaging flow cytometry for the study of erythroid cell biology and pathology. J Immunol Methods 2015;423:52-9

55. Kviatkovsky I, Zeidan A, Yeheskely-Hayon D, Shabad EL, Dann EJ, Yelin D. Measuring sickle cell morphology during blood flow. Biomed Opt Express 2017;8:1996-2003

56. van Beers EJ, Samsel L, Mendelsohn L, Saiyed R, Fertrin KY, Brantner CA, Daniels MP, Nichols J, McCoy JP, Kato GJ. Imaging flow cytometry for automated detection of hypoxia-induced erythrocyte shape change in sickle cell disease. Am J Hematol 2014;89:598-603

57. Fertrin KY, van Beers EJ, Samsel L, Mendelsohn LG, Saiyed R, Nichols JS, Hepp DA, Brantner CA, Daniels MP, McCoy JP, Kato GJ. Imaging flow cytometry documents incomplete resistance of human sickle Fcells to ex vivo hypoxia-induced sickling. Blood 2014;124:658-60

58. Villarreal N, Lu M, Shevkoplyas SS. A supplement to TRANSFUSION abstract presentations from the AABB annual meeting San Antonio, TX, October 19-22, 2019. Transfusion 2019;59:8A-220A

59. Xu M, Papageorgiou DP, Abidi SZ, Dao M, Zhao H, Karniadakis GE. A deep convolutional neural network for classification of red blood cells in sickle cell anemia. PLoS Comput Biol 2017;13:e1005746

60. Nassar M, Doan M, Filby A, Wolkenhauer O, Fogg DK, Piasecka J, Thornton CA, Carpenter AE, Summers HD, Rees P. Label-free identification of white blood cells using machine learning. Cytometry Part A 2019;95:836-842

61. Piety NZ, Gifford SC, Yang X, Shevkoplyas SS. Quantifying morphological heterogeneity: a study of more than 1000000 individual stored red blood cells. Vox Sang 2015;109:221-30

62. Di Liberto G, Kiger L, Marden MC, Boyer L, Poitrine FC, Conti M, Rakotoson MG, Habibi A, Khorgami S, Vingert B, Maitre B, Galacteros F, Pirenne F, Bartolucci P. Dense red blood cell and oxygen desaturation in sickle-cell disease. Am J Hematol 2016;91:1008-13

63. Koren A, Segal-Kupershmit D, Zalman L, Levin C, Abu Hana M, Palmor H, Luder A, Attias D. Effect of hydroxyurea in sickle cell anemia: a clinical trial in children and teenagers with severe sickle cell anemia and sickle cell beta-thalassemia. Pediatr Hematol Oncol 1999;16:221-32

64. Cieri-Hutcherson NE, Hutcherson TC, Conway-Habes EE, Burns BN, White NA. Systematic review of L-glutamine for prevention of vasoocclusive pain crisis in patients with sickle cell disease. Pharmacotherapy 2019;39:1095-104

65. Borba R, Lima CS, Grotto HZ. Reticulocyte parameters and hemoglobin $\mathrm{F}$ production in sickle cell disease patients undergoing hydroxyurea therapy. J Clin Lab Anal 2003;17:66-72

66. Penkert RR, Hurwitz JL, Thomas P, Rosch J, Dowdy J, Sun Y, Tang L, Hankins JS. Inflammatory molecule reduction with hydroxyurea therapy in children with sickle cell anemia. Haematologica 2018;103:e50-e54

67. Bartolucci P, Chaar V, Picot J, Bachir D, Habibi A, Fauroux C, Galacteros F, Colin Y, Le Van Kim C, El Nemer W. Decreased sickle red blood cell adhesion to laminin by hydroxyurea is associated with inhibition of Lu/BCAM protein phosphorylation. Blood 2010;116:2152-9

68. Cartron JP, Elion J. Erythroid adhesion molecules in sickle cell disease: effect of hydroxyurea. Transfus Clin Biol 2008;15:39-50 
69. Goldberg MA, Brugnara C, Dover GJ, Schapira L, Lacroix L, Bunn HF. Hydroxyurea and erythropoietin therapy in sickle cell anemia. Semin Oncol 1992;19:74-81

70. Niihara Y, Miller ST, Kanter J, Lanzkron S, Smith WR, Hsu LL, Gordeuk VR, Viswanathan K, Sarnaik S, Osunkwo I. A phase 3 trial of L-glutamine in sickle cell disease. N Engl J Med 2018;379:226-35

71. Quinn CT. L-glutamine for sickle cell anemia: more questions than answers. Blood 2018;132:689-93

72. Robinson TM, Fuchs EJ. Allogeneic stem cell transplantation for sickle cell disease. Curr Opin Hematol 2016;23:524-9

73. Shenoy S. Hematopoietic stem-cell transplantation for sickle cell disease: current evidence and opinions. Ther Adv Hematol 2013;4:335-44

74. Brousse V, Buffet P, Rees D. The spleen and sickle cell disease: the sick (led) spleen. Br J Haematol 2014;166:165-76

75. Oder E, Safo MK, Abdulmalik O, Kato GJ. New developments in antisickling agents: can drugs directly prevent the polymerization of sickle haemoglobin in vivo? Br J Haematol 2016;175:24-30

76. Howard J, Hemmaway CJ, Telfer P, Layton DM, Porter J, Awogbade M, Mant T, Gretler DD, Dufu K, Hutchaleelaha A, Patel M, Siu V, Dixon S, Landsman N, Tonda M, Lehrer-Graiwer J. A phase 1/2 ascending dose study and open-label extension study of voxelotor in patients with sickle cell disease. Blood 2019;133:1865-75

77. Vichinsky E, Hoppe CC, Ataga KI, Ware RE, Nduba V, El-Beshlawy A, Hassab H, Achebe MM, Alkindi S, Brown RC, Diuguid DL, Telfer P, Tsitsikas DA, Elghandour A, Gordeuk VR, Kanter J, Abboud MR, Lehrer-Graiwer J, Tonda M, Intondi A, Tong B, Howard J, Investigators HT. A phase 3 randomized trial of voxelotor in sickle cell disease. N Engl J Med 2019;381:509-19
78. Telfer P, Agodoa I, Fox KM, Burke L, Mant T, Jurek M, Tonda M, LehrerGraiwer J. Impact of voxelotor (GBT440) on unconjugated bilirubin and jaundice in sickle cell disease. Hematol Rep 2018;10:e7643

79. Dufu K, Patel M, Oksenberg D, Cabrales P. GBT440 improves red blood cell deformability and reduces viscosity of sickle cell blood under deoxygenated conditions. Clin Hemorheol Microcirc 2018;70:95-105

80. Hutchaleelaha A, Patel M, Washington C, Siu V, Allen E, Oksenberg D, Gretler DD, Mant T, Lehrer-Graiwer J. Pharmacokinetics and pharmacodynamics of voxelotor (GBT440) in healthy adults and patients with sickle cell disease. Br J Clin Pharmacol 2019;85:1290-302

81. Shimp EA, Alsmadi NZ, Cheng T, Lam KH, Lewis CS, Schmidtke DW Effects of shear on P-selectin deposition in microfluidic channels. Biomicrouidics 2016;10:024128

82. Poletti V, Urbinati F, Charrier S, Corre G, Hollis RP, Fernandez BC, Martin S, Rothe M, Schambach A, Kohn DB. Pre-clinical development of a lentiviral vector expressing the anti-sickling $\beta$ AS3 globin for gene therapy for sickle cell disease. Mol Ther Methods Clin Dev 2018;11:167-79

83. Cavazzana M, Ribeil J-A, Payen E, Touzot F, Neven B, Lefrere F, Suarez F, Magrin E, Beuzard Y, Chretien S. 279. Clinical outcomes of gene therapy with BB305 lentiviral vector for sickle cell disease and $\beta$-Thalassemia. Molecular Therapy 2016;24:S111-S12

84. Lin MI, Paik E, Mishra B, Burkhardt D, Kernytsky A, Pettiglio M, Chen Y-S, Tomkinson K, Woo A, Cortes M. CRISPR/Cas9 genome editing to treat sickle cell disease and B-Thalassemia: re-creating genetic variants to upregulate fetal hemoglobin appear well-tolerated, effective and durable. Am Soc Hematology 2017;130:10661-5

85. Connes P, Hue O, Tripette J, Hardy-Dessources MD. Blood rheology abnormalities and vascular cell adhesion mechanisms in sickle cell trait carriers during exercise. Clin Hemorheol Microcirc 2008;39:179-84 